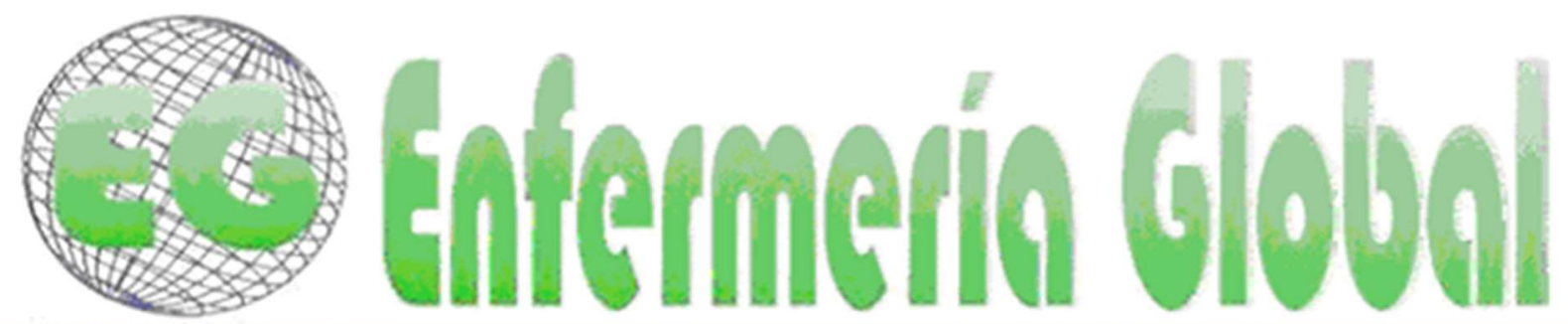

\title{
Temperatura corporal y temperatura de calentamiento en el cuidado de pacientes grandes quemados
}

Body temperature and heating temperature in major burns patients care

\author{
Juan Manuel Alonso Fernández ${ }^{1}$ \\ Carlos López Pablo ${ }^{2}$
}

\begin{abstract}
1 Máster en Investigación en Ciencias de la Enfermería. Enfermero Unidad de Cuidados Intensivos. Hospital Universitario Rio Hortega. Valladolid. España. jualonsofern@saludcastillayleon.es

2 Docente. Máster en Investigación en Ciencias de la Enfermería. Universidad Rovira i Virgili. Tarragona. España.
\end{abstract}

\section{https://doi.org/10.6018/eglobal.430221}

Recibido: 31/05/2020

Aceptado: $25 / 09 / 2020$

\section{RESUMEN:}

Objetivos: El objetivo es describir los métodos de calentamiento y su aplicación para el mantenimiento de la temperatura corporal en el paciente gran quemado.

Metodología: Revisión bibliográfica realizada entre septiembre de 2019 y febrero de 2020 acerca de la termorregulación y calentamiento del paciente quemado en las bases de datos CINAHL, CUIDEN, PUBMED, MEDES y WOS en español e inglés, de los últimos 10 años, de los cuales fueron analizados 24 documentos.

Resultados: La respuesta hipermetabólica y la reprogramación hipotalámica provocan un aumento de la temperatura basal en los pacientes quemados, entre 37 y $38,5^{\circ} \mathrm{C}$ sin origen infeccioso. Para disminuir el gasto energético en reposo y la repuesta hipermetabólica se puede aplicar una temperatura ambiental elevada, como calentamiento externo pasivo, entre 28 y $32^{\circ} \mathrm{C}$.

Discusión: Existen otros métodos de calentamiento externo activo que pueden conseguir el mismo objetivo como las mantas de aire convectivo, placas térmicas o sistemas de superficie.

Conclusión: Debe revisarse la recomendación de calentamiento mediante temperatura ambiental elevada, que crea ambientes hostiles para los trabajadores y los pacientes, a través del estudio de la inclusión de métodos de calentamiento externo activo.

Palabras clave: Quemados; Regulación de la Temperatura Corporal; Calentadores; Metabolismo energético; Atención de Enfermería.

\section{ABSTRACT:}

Objectives: To describe the heating methods and their application to maintain body temperature in majors burn patients.

Methodology: Bibliographic review carried out between September 2019 and February 2020 about the thermoregulation and heating of the burn patient in the CINHAL, CUIDEN, PUBMED, MEDES and WOS databases in Spanish and English, documents from the last 10 years, from which 26 were analyzed.

Results: Hypermetabolic response and hypothalamic reprogramming cause an increase in basal temperature in burn patients between 37 and $38.5^{\circ} \mathrm{C}$ without infectious origin. To decrease the energy 
expenditure at rest and the hypermetabolic response, it is possible to act through a high ambient temperature between 28 and $32^{\circ} \mathrm{C}$ as passive external heating.

Discussion: Other external heating methods can achieve this goal such as convective air blankets, heating plates, or surface systems.

Conclusions: The recommendation of warming by means of high ambient temperature, which creates hostile environments for workers and patients, should be reviewed through the study of the inclusion of active external warming methods.

Key words: Burn patient; Body Temperature Regulation; Heating; Energy metabolism; Nursing Care.

\section{INTRODUCCIÓN}

La piel es el órgano más extenso del organismo, realiza funciones de mantenimiento de la homeostasis y regulación térmica. La pérdida de la piel por una quemadura precisará el reemplazo temporal de estas funciones hasta su recuperación. La OMS define las quemaduras como un problema de salud mundial, con una prevalencia 7 veces mayor en países en desarrollo, producidas fundamentalmente en el ámbito doméstico, siendo la prevención la intervención que más puede reducir la mortalidad ${ }^{(1)}$.

En España, se produjeron 1.757 muertes por accidentes de fuego, humo y sustancias calientes, en la última década, 1996-2016, con una tendencia al alza desde el año 2011. El año 2017 se registraron 9.186 ingresos por quemaduras con una incidencia fue de 7 casos por cada 100.000 habitantes y con un total de 39.170 estancias hospitalarias. European Burns Association (EBA) describe que 14 de cada 100.000 personas precisarán atención hospitalaria por quemaduras al año ${ }^{(2)}$.

Estos pacientes presentan habitualmente hipotermia tras sufrir la quemadura y cuando se trasladan a una unidad de quemados reciben unos cuidados más especializados ${ }^{(3)}$. Las quemaduras con una extensión mayor de $20 \%$ de Superficie Corporal Quemada (SCQ), edad avanzada y otras morbilidades aumentan el riesgo de sufrir hipotermia.

El riesgo de desarrollar hipotermia en las primeras 72 horas vendrá determinado por un lado por la respuesta fisiopatológica, la extensión de la quemadura, la exposición ambiental y el flujo aéreo; y por otra parte debido a la actuación sanitaria sobre el paciente. Se relaciona la hipotermia con coagulopatía y elevado gasto calórico ${ }^{(4)}$. Entre un $40-80 \%$ de los pacientes que sufren quemaduras graves presentan hipotermia durante la fase aguda $(3,5,6)$, relacionándose directamente con la extensión de la quemadura. La hipotermia tiene mayor prevalencia en edad avanzada, quemaduras mayores al $40 \%$ SCQ y en sexo femenino ${ }^{(5-7)}$. Además, se describe la presencia de hipotermia como factor independiente que puede aumentar hasta un $5 \%$ la mortalidad de estos pacientes ${ }^{(5)}$.

Desde que se produce el accidente que provoca la quemadura se inicia el proceso de atención del paciente gran quemado. La enfermera tiene la competencia para la emisión del diagnóstico hipotermia o riesgo de hipotermia, estableciendo un plan de cuidados, y define como objetivo la normotermia. Se deben reajustar los mecanismos de calentamiento para lograr la temperatura destino, consiguiendo un equilibrio entre la producción, la ganancia y la pérdida de calor.

El objetivo es describir los métodos de calentamiento y su aplicación para el mantenimiento de la temperatura corporal en el paciente gran quemado. 


\section{MATERIAL Y MÉTODOS}

Se realizó una revisión bibliográfica entre septiembre de 2019 y febrero de 2020, acerca de la regulación térmica, el calentamiento en la hipotermia y control metabólico en pacientes quemados con los términos de búsqueda, DeCS y MeSH: Quemaduras Burns, Quemados - Burns patient, Hipotermia - Hypothermia, Temperatura corporal, Body temperature, Regulación de la Temperatura Corporal- Body Temperature Regulation, Calefacción - Heating, Calentadores-Heaters, Metabolismo energético Energy metabolism, Consumo de energía - Energy consumption, Metabolismo basal Basal metabolism, Estrés fisiológico - Stress,Physiological, Temperatura ambiental Temperature, Temperatura corporal - Body temperature, Atención de Enfermería Nursing Care, , Enfermera - Nurses, Enfermería - Nursing, Unidades de QuemadosBurn Units, Confort térmico-Thermal Comfort, en las bases de datos: CINHAL, CUIDEN, PUBMED, MEDES, el metabuscador Web Of Sciencie (WOS), en las bases de datos secundarias: biblioteca Cochrane, Joanna Briggs Connect, UptoDate, GuiaSalud; y en la páginas de referencias de sociedades internacionales de atención a pacientes con quemaduras ISBI, ABA y EBA. También se incluyó la búsqueda de literatura gris, tesis doctorales en TESEO y otros documentos de referencia relacionados. Se incluyeron artículos en español e inglés, publicados desde 2009, rango necesario para la definición adecuada de los conceptos que se revisan de pacientes adultos con quemaduras térmicas, y contenido relacionado con cuidados del paciente con quemaduras, su calentamiento, termorregulación o metabolismo. Se excluyeron documentos de quemaduras de pequeña extensión, por quemaduras químicas o eléctricas, con lesión por inhalación, en atención extrahospitalaria o de la fase post aguda.

\section{RESULTADOS}

Se encontraron 3536 artículos, según las bases de datos y motores de búsqueda consultados, aplicando los filtros de búsqueda con los términos definidos anteriormente fueron obtenidos 199 que tras el cribado y selección para su posterior evaluación y análisis resultaron 24 documentos. Se exponen los antecedentes y revisión del tema de investigación a continuación categorizados por apartados.

\section{Temperatura corporal en paciente gran quemado}

La pérdida de la superficie de la piel por una quemadura lleva directamente a la pérdida de sus funciones produciéndose una alteración en la termorregulación, alteración de la regulación del sudor (pérdidas de calor por evaporación) y alteración de la regulación del flujo sanguíneo (pérdidas de calor por radiación y convección) ${ }^{(8)}$.

Las pérdidas de calor del paciente quemado se presentan por conducción, convección, radiación y evaporación ${ }^{(9)}$. El control de las pérdidas por conducción se realizará actuando sobre la superficie que se posicione al paciente ya sea la camilla de transporte, cama o mesa quirúrgica. El tratamiento sobre las pérdidas por convección se actuará controlando la temperatura y humedad ambientales y protegiendo de las corrientes de aire. Las pérdidas por radiación serán las pérdidas hacia el entorno, por lo que debe limitarse la exposición corporal del paciente. $Y$ frente a las pérdidas por evaporación se deberá limitar el contacto con apósitos húmedos y el control de la 
sudoración ${ }^{(9)}$. Para paliar estas pérdidas se aplicarán medidas de calentamiento y mantenimiento de la temperatura.

La pérdida de calor y agua a través de la quemadura provoca un aumento de temperatura central, reprogramando la regulación hipotalámica, por lo que se espera que los pacientes presenten una temperatura superior de hasta $2^{\circ} \mathrm{C}$ sin foco infeccioso situándose en torno a los $38,5^{\circ} \mathrm{C}(10)$. Debe evitarse, por sus efectos indeseados, una temperatura corporal inferior a $35^{\circ} \mathrm{C}(11)$ y a pesar de desconocer la temperatura diana óptima, ésta debería ser mayor de $37,5^{\circ} \mathrm{C}{ }^{(4)}$.

Figura 1: Pérdidas de calor del paciente quemado. Elaboración propia.

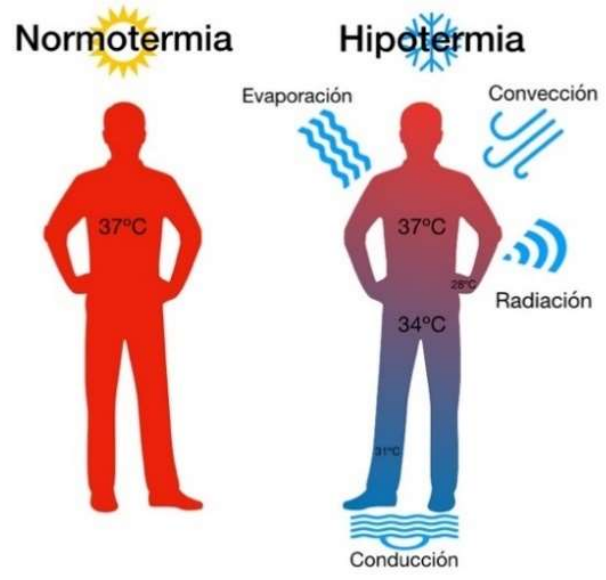

La hipotermia en pacientes quemados puede aumentar las lesiones por la vasoconstricción, provocar alteraciones de la coagulación, disminución de la actividad enzimática, alteración de la respiración, pudiendo ocasionar arritmias y muerte ${ }^{(11-13)}$. La atención centralizada ofrecida en una unidad de grandes quemados proporciona un entorno controlado y estandarizado indispensable para supervivencia de estos pacientes críticos e inmunocomprometidos ${ }^{(14)}$.

La actuación prehospitalaria y la aplicación de las recomendaciones establecidas para el enfriamiento de la quemadura, controlando el riesgo de hipotermia y aplicando las medidas de control de pérdida de calor, determinarán la progresión de la hipotermia y la necesidad de un calentamiento más rápido de los pacientes con quemaduras extensas ${ }^{(3,6)}$.

Una vez estabilizado y durante las primeras horas después de la lesión, el paciente necesitará ser cubierto con mantas limpias y secas para evitar o limitar la hipotermia. Entre las medidas del mantenimiento de la temperatura corporal, está la cura oclusiva para evitar las pérdidas de calor, la infección local y promover la curación. El efecto barrera de los apósitos asegura el aislamiento térmico y mantenimiento de la humedad, permitiendo el intercambio gaseoso. Junto a la limitación y control de la exposición corporal, son factores relevantes para evitar la disminución de la temperatura corporal ${ }^{(12)}$.

Se denomina temperatura crítica a la temperatura que desencadena una respuesta celular termogénica. A medida que es mayor la gravedad de la quemadura, la temperatura crítica disminuye. Si bien la temperatura crítica y el tiempo en alcanzarla 
puede variar entre pacientes, se puede controlar la respuesta hipermetabólica mediante temperatura ambiental elevada, minimizando las pérdidas por calor, mejorando la coagulación y disminuyendo la morbilidad y la mortalidad (4).

El rango de temperaturas destino de los pacientes en un estudio en unidades de quemados de EEUU fue entre $36-38^{\circ} \mathrm{C}{ }^{(15)}$. A pesar de no existir una definición clara de la temperatura destino, se encuentra relación entre mayor temperatura corporal y menor gasto metabólico. Se considerará hipotermia con temperatura corporal menor de $36,5^{\circ} \mathrm{C}$ en el paciente quemado (Tabla 1 ).

Tabla 1: Temperatura corporal en pacientes grandes quemados

\begin{tabular}{|c|c|c|c|}
\hline Autores & Metodología / Muestra & Evento & Recomendación \\
\hline $\begin{array}{l}\text { Sommerhalder } \\
\text { et al, } 2020\end{array}$ & $\begin{array}{l}\text { Revisión problemas } \\
\text { actuales en } \\
\text { hipermetabolismo en } \\
\text { quemados }\end{array}$ & $\begin{array}{l}\text { En base al estudio de } \\
\text { Corallo, no existe un guía } \\
\text { clínica sobre el manejo de la } \\
\text { temperatura }\end{array}$ & $\begin{array}{l}\text { Mantener una } \\
\text { temperatura en torno a } \\
37,5^{\circ} \mathrm{C}\end{array}$ \\
\hline $\begin{array}{l}\text { Alonso- } \\
\text { Fernández et al, } \\
2019\end{array}$ & $\begin{array}{l}\text { Estudio observacional } \\
\text { retrospectivo de } \\
\text { temperatura en } 58 \\
\text { pacientes en las primeras } \\
72 \text { horas }\end{array}$ & $\begin{array}{l}\text { Ingreso en Unidad de } \\
\text { Quemados en hipotermia, } \\
\text { menos de } 35^{\circ} \mathrm{C} \text { Alcanzar } \\
\text { normotermia pasadas } 16 \\
\text { horas. }\end{array}$ & $\begin{array}{l}\text { Mayor esfuerzo en el } \\
\text { recalentamiento. } \\
\text { Temperatura media de } \\
37^{\circ} \mathrm{C} \text {, aumentar } \\
\text { medidas de } \\
\text { calentamiento }\end{array}$ \\
\hline $\begin{array}{l}\text { Pahm et al, } \\
2018\end{array}$ & $\begin{array}{l}\text { Revisión de pautas de } \\
\text { tratamiento en pacientes } \\
\text { quemados en atención } \\
\text { inicial }\end{array}$ & $\begin{array}{l}\text { En caso de hipotermia y No } \\
\text { comenzar a recalentar } \\
\text { extremidades hasta alcanzar } \\
35^{\circ} \mathrm{C} \text { de temperatura central } \\
\text { para evitar una caída de } \\
\text { temperatura }\end{array}$ & $\begin{array}{l}\text { Evitar temperatura } \\
\text { menor de } 35^{\circ} \mathrm{C}\end{array}$ \\
\hline Gaickwad, 2016 & Revisión sistemática & $\begin{array}{l}\text { Pérdidas de calor del } \\
\text { paciente quemado por } \\
\text { conducción, convección, } \\
\text { radiación y evaporación }\end{array}$ & $\begin{array}{l}\text { Limitar la exposición } \\
\text { corporal del paciente } \\
\text { para evitar la } \\
\text { hipotermia. }\end{array}$ \\
\hline $\begin{array}{l}\text { Weaver et al, } \\
2014\end{array}$ & $\begin{array}{l}\text { Estudio descriptivo } \\
\text { retrospectivo de ingreso } \\
\text { de } 2270 \text { pacientes } \\
\text { quemado }\end{array}$ & $\begin{array}{l}\text { Temperatura de ingreso de } \\
36,7^{\circ} \mathrm{C}, 3,5 \% \text { con } \\
\text { temperatura menor de } 35^{\circ} \mathrm{C}\end{array}$ & $\begin{array}{l}\text { Definición de } \\
\text { hipotermia entre } 34^{\circ} \mathrm{C} \text {, } \\
35^{\circ} \mathrm{C} \text { y } 36^{\circ} \mathrm{C} \text {. } \\
\text { Aumentar esfuerzos } \\
\text { en aumentar la } \\
\text { temperatura corporal } \\
\text { al ingreso. }\end{array}$ \\
\hline $\begin{array}{l}\text { Hostler et al, } \\
2013\end{array}$ & $\begin{array}{l}\text { Estudio descriptivo } \\
\text { retrospectivo de } 12097 \\
\text { pacientes con } \\
\text { quemaduras }\end{array}$ & $\begin{array}{l}\text { Hipotermia asociada a } \\
\text { quemaduras mayores al } \\
20 \% \text { de SCQ, edad } \\
\text { avanzada. Alto índice de } \\
\text { masa corporal protector de } \\
\text { la hipotermia }\end{array}$ & $\begin{array}{l}\text { Define hipotermia } \\
\text { temperatura menor de } \\
36,5^{\circ} \mathrm{C}\end{array}$ \\
\hline $\begin{array}{l}\text { Bishop et al, } \\
2012\end{array}$ & $\begin{array}{l}\text { Revisión cuidado crítico } \\
\text { quemados, } 7 \text { referencias }\end{array}$ & $\begin{array}{l}\text { Temperatura base central } \\
\text { en pacientes grandes } \\
\text { quemados } 38,5^{\circ} \mathrm{C}\end{array}$ & $\begin{array}{l}\text { Procurar temperatura } \\
\text { mayor de } 37,5^{\circ} \mathrm{C} \text {. } \\
\text { Temperatura de } 37^{\circ} \mathrm{C} \\
\text { se considera } \\
\text { hipotermia relativa }\end{array}$ \\
\hline $\begin{array}{l}\text { Willmore et al, } \\
1975\end{array}$ & $\begin{array}{l}\text { Estudio de casos y } \\
\text { controles, } 8 \text { pacientes } \\
\text { quemados, } 4 \text { casos } \\
\text { control }\end{array}$ & $\begin{array}{l}\text { Tasa metabólica calculada } \\
\text { mediante el consumo de } \mathrm{O}_{2} \\
\text { exhalado, pérdidas } \\
\text { insensibles de } \mathrm{H}_{2} \mathrm{O} \text { a } 25^{\circ} \mathrm{C} \text { y } \\
33^{\circ} \mathrm{C}\end{array}$ & $\begin{array}{l}\text { Temperatura mayor de } \\
1,7^{\circ} \mathrm{C}-2^{\circ} \mathrm{C} \text { en } \\
\text { pacientes quemados. }\end{array}$ \\
\hline
\end{tabular}

*GMR Gasto Metabólico en Reposo, ** SCQ Superficie Corporal Quemada

En el cuidado del paciente quemado, se debe tener en cuenta que la temperatura basal de estos pacientes es de $38,5^{\circ} \mathrm{C}$, y que una temperatura menor de $37^{\circ} \mathrm{C}$ puede considerarse relativamente como hipotermia ${ }^{(16)}$. 


\section{Metabolismo energético en pacientes quemados}

Se consideran 2 fases en la respuesta metabólica del paciente quemado, la fase aguda que va desde el accidente hasta las 72 horas, y la fase hipermetabólica. El estado hipermetabólico que desarrolla el paciente quemado tras la fase aguda aumenta el consumo energético. Son necesarias varias actuaciones para disminuir los efectos de hipermetabolismo para reducir el gasto calórico, al menos para que no se produzca un aumento del metabolismo ${ }^{(17) .}$

Quemaduras mayores al 20\% de SCQ desarrollarán una respuesta hipermetabólica, inflamatoria y de estrés de manera similar a la que se desarrollan con quemaduras superiores al $40 \%$ de SCQ ${ }^{(18)}$. Se recomienda actuar sobre hipermetabolismo, ya que su disminución mejorará la curación de las quemaduras. El aporte nutricional precoz y adecuado al paciente intervendrá sobre el estado catabólico disminuyendo la demanda energética ${ }^{(19)}$.

Durante la fase aguda tras la quemadura, el paciente tiene una pérdida severa de masa corporal magra, desequilibrio de radicales libres en relación con los mecanismos de defensa antioxidante y deterioro de la función inmune. La intervención mediante un soporte nutricional adecuado y el inicio precoz de nutrición enteral satisfarán las demandas energéticas de proteínas, grasas, lípidos y micronutrientes. Además, el suplemento de micronutrientes como la glutamina, contribuirán a la recuperación de la masa magra ${ }^{(20)}$.

El gasto metabólico de los pacientes quemados en el estudio de Stanojcic et al ${ }^{(18)}$ fue de 1,4 $\pm 0,2$ veces el gasto metabólico basal mientras que en el estudio de Leung et al(21) fue de $1,43 \pm 0,32$ veces el GMB en pacientes con quemaduras menores del $40 \%$. Se encuentran pérdidas hídricas de hasta $4 \mathrm{l} / \mathrm{m}^{2}$ de superficie quemada al día ${ }^{(22)}$. Las pérdidas de líquidos corporales por evaporación que se cuantifican son de 3 a 4 veces mayor que en la piel sana según sean quemaduras de segundo grado profundo y tercer grado. Puede ser valorado mediante el índice de pérdida transepidérmica de agua (TEWL), midiendo la cantidad de agua en gramos que pasa a través de la piel a la atmósfera circundante, por hora y área de piel, siendo según la profundidad de la quemadura de 50 a $62 \mathrm{gr} / \mathrm{m}^{2}$ a la hora ${ }^{(23)}$ (Tabla 2).

Tabla 2: Termorregulación en pacientes grandes quemados

\begin{tabular}{|c|c|c|c|c|}
\hline & Autores & $\begin{array}{l}\text { Metodología } \\
\text { Muestra }\end{array}$ & Evento & Conclusión \\
\hline \multirow{2}{*}{ 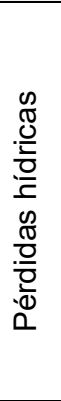 } & $\begin{array}{l}\text { Busche et al, } \\
2016\end{array}$ & $\begin{array}{l}\text { Estudio de pérdidas } \\
\text { hídricas en } 28 \\
\text { pacientes }\end{array}$ & $\begin{array}{l}50 \text { a } 62 \mathrm{~g} / \mathrm{m} 2 \text { a la hora. } \\
\text { Presenta diferencias } \\
\text { entre quemaduras, } \\
\text { zonas donantes y } \\
\text { cobertura con sustitutos } \\
\text { biotesintéticos. }\end{array}$ & $\begin{array}{l}\text { Perdías hídricas } 3 \text { veces mayor } \\
\text { en quemaduras profundas y } 4 \\
\text { veces mayor en quemaduras } \\
\text { superficiales }\end{array}$ \\
\hline & $\begin{array}{l}\text { Zacwaki et al, } \\
1970\end{array}$ & $\begin{array}{l}\text { Estudio de } 20 \\
\text { pacientes } \\
\text { consumo de } \mathrm{O}_{2} \text { y } \\
\text { pérdidas insensibles } \\
\text { de agua }\end{array}$ & $\begin{array}{l}\text { Pérdidas de } 4 \mathrm{l} / \mathrm{m} 2 \text {. } \\
\text { La exposición de la } \\
\text { quemadura presenta } \\
\text { pérdidas de } 0,5^{\circ} \mathrm{F}\end{array}$ & $\begin{array}{l}\text { El hipermetabolismo aumenta } \\
\text { las pérdidas hídricas. } \\
\text { La disminución de la } \\
\text { exposición corporal disminuye } \\
\text { las pérdidas }\end{array}$ \\
\hline \multirow[t]{2}{*}{$\begin{array}{l} \\
0 \\
0 \\
0 \\
0 \\
0 \\
0 \\
0\end{array}$} & $\begin{array}{l}\text { Sommerhalder } \\
\text { et al, } 2020\end{array}$ & $\begin{array}{l}\text { Revisión problemas } \\
\text { actuales en } \\
\text { hipermetabolismo } \\
\text { en quemados }\end{array}$ & $\begin{array}{l}\text { A mayor SCQ desde el } \\
20 \% \text { mayor incremento } \\
\text { del GMB }\end{array}$ & $\begin{array}{l}\text { Aumento progresivo del GMR } \\
\text { dependiente de la temperatura } \\
\text { ambiental, basado en el } \\
\text { estudio de Kelemen }\end{array}$ \\
\hline & $\begin{array}{l}\text { Leung et al, } \\
2019\end{array}$ & $\begin{array}{l}\text { Estudio } \\
\text { observacional }\end{array}$ & $\begin{array}{l}\text { Presentaron una ratio } \\
\text { de GMR } 1,43 \pm 0,32\end{array}$ & $\begin{array}{l}\text { Recomendación de utilización } \\
\text { de calorimetría indirecta para }\end{array}$ \\
\hline
\end{tabular}




\begin{tabular}{|c|c|c|c|}
\hline & $\begin{array}{l}\text { retrospectivo. } \\
\text { Medida de Gasto } \\
\text { Energético en } 29 \\
\text { pacientes }\end{array}$ & $\begin{array}{ll}\text { Diferencia entre } & 4 \\
\text { escalas predictivas } & \text { de } \\
\text { GMB. incluye } & \\
\text { No la } \\
\text { temperatura en el } \\
\text { estudio }\end{array}$ & $\begin{array}{l}\text { calcular el Gasto Metabólico en } \\
\text { Reposo para el control del } \\
\text { hipermetabolismo del paciente } \\
\text { quemado. }\end{array}$ \\
\hline $\begin{array}{l}\text { Stanojcic et al, } \\
2018\end{array}$ & $\begin{array}{l}\text { Estudio } \\
\text { retrospectivo del } \\
\text { Gasto metabólico en } \\
1288 \text { pacientes }\end{array}$ & $\begin{array}{l}\text { Presentaron una ratio } \\
\text { de GMR } 1,4 \pm 0,2 \\
\text { calculado mediante un } \\
\text { intercambiador } \\
\text { metabólico. } \\
\begin{array}{ll}\text { considera temperatura } \\
\text { corporal }\end{array}\end{array}$ & $\begin{array}{l}\text { Control de aumento del } \\
\text { hipermetabolismo, respuesta } \\
\text { inflamatoria y estrés en } \\
\text { quemados con más del } 20 \% \text { de } \\
\text { SCQ }\end{array}$ \\
\hline
\end{tabular}

*GMR Gasto Metabólico en Reposo, ** SCQ Superficie Corporal Quemada

\section{Métodos de calentamiento}

Las medidas para paliar las pérdidas de calor deberán actuar en el calentamiento y mantenimiento de la temperatura corporal del paciente a través de métodos de calentamiento interno activo como la administración de líquidos calientes vía intravenosa y otros métodos de calentamiento externo que serán pasivos y/o activos.

Un análisis sobre la utilización de los métodos de calentamiento, en unidades de quemados de EEUU, declararon en relación con el total de casos: el aumento de la temperatura ambiental el $94,2 \%$, seguido de aparatos de aire forzado el $88,5 \%$, líquidos intravenosos templados el $82,7 \%$, almohadillas calefactoras conductoras el $38,5 \%$ y catéter intravascular con regulación de temperatura el 21,6\%(15) (Tabla 3).

\section{Calentamiento externo pasivo: Temperatura ambiental}

Como método de calentamiento externo pasivo se utiliza una temperatura ambiental elevada, las recomendaciones la sitúan entre 28 y $33^{\circ}{ }^{(11)}$. Este aumento de temperatura ambiental puede reducir de 2,0 veces a 1,4 el Gasto Energético en Reposo en pacientes con quemaduras mayores al $40 \%$ SCQ.

Esta medida sirve para minimizar el aumento de la tasa metabólica basal causada por la generación de calor y la pérdida de agua por evaporación. Es una recomendación Grado B de evidencia por Joanna Briggs Institute ${ }^{(24)}$, proporcionar una habitación cálida con temperatura ambiental de $28-32^{\circ} \mathrm{C}$, para prevenir la hipotermia.

Pruskowski et al observaron que el aumento de la temperatura ambiental era el calentamiento más usado en unidades de quemados, utilizando un amplio arco de temperatura de 24 a $35^{\circ} \mathrm{C}{ }^{(15)}$.

Tabla 3: Temperatura ambiental en el cuidado de pacientes grandes quemados

\begin{tabular}{|c|c|c|c|}
\hline Autores & $\begin{array}{l}\text { Tipo de estudio I } \\
\text { Muestra }\end{array}$ & Variables & Resultados \\
\hline $\begin{array}{l}\text { Sommerhalder } \\
\text { et al, } 2020\end{array}$ & $\begin{array}{l}\text { Recomendación } \\
\text { experta }\end{array}$ & $\begin{array}{l}\text { Estudio de Kelemen, } \\
\text { disminución del GMR un } \\
22^{\circ} \mathrm{C} \text { con temperatura } \\
\text { ambiental } 32^{\circ} \mathrm{C} \text { frete a } \\
22^{\circ} \mathrm{C}\end{array}$ & $\begin{array}{l}\text { Temperatura habitación UCI } 28 \\
-30^{\circ} \mathrm{C}\end{array}$ \\
\hline \multirow[t]{2}{*}{$\begin{array}{l}\text { Pruskowski et } \\
\text { al, } 2018\end{array}$} & \multirow{2}{*}{ 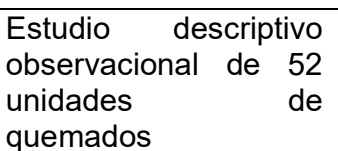 } & $\begin{array}{l}\text { Temperatura en fase de } \\
\text { Resucitación }\end{array}$ & $\begin{array}{l}67,3 \% \text { UCl's a } 75-80^{\circ} \mathrm{F} \\
38,5 \% \text { UCl's a } 90-95^{\circ} \mathrm{F}\end{array}$ \\
\hline & & $\begin{array}{l}\text { Temperatura } \\
\text { Habitación }\end{array}$ & $\begin{array}{l}67,3 \% \text {.UCl's a } 75-80^{\circ} \mathrm{F} \\
40,3 \% \text { UCl's a } 85-90^{\circ} \mathrm{F}\end{array}$ \\
\hline
\end{tabular}




\begin{tabular}{|c|c|c|c|}
\hline & & $\begin{array}{l}\text { Temperatura ambiental } \\
\text { Durante las curas }\end{array}$ & $\begin{array}{l}63,5 \% \text { UCl's a } 75-80^{\circ} \mathrm{F} \\
42,3 \% \text { UCl's a } 85 .-90^{\circ} \mathrm{F}\end{array}$ \\
\hline \multirow[t]{2}{*}{$\begin{array}{l}\text { Kelemen et al, } \\
1996\end{array}$} & $\begin{array}{l}\text { Estudio de casos y } \\
\text { controles con } 44 \\
\text { pacientes quemados y } \\
4 \text { controles }\end{array}$ & \multirow{2}{*}{$\begin{array}{l}\text { Correlación negativa entre } \\
\text { la temperatura de } 22 \text { a } \\
32^{\circ} \mathrm{C} \text { y la tasa de } \\
\text { GMR/GMB. } \\
\text { Establece que la reducción } \\
\text { dependiente de la } \\
\text { temperatura ambiental va } \\
\text { de } 2,25 \text { a } 1,5 \text { la tasa } \\
\text { GMR/GMB }\end{array}$} & $\begin{array}{l}\text { El grupo control no encontraron } \\
\text { diferencias significativas en el } \\
\text { ratio GMR/GMB a diferentes } \\
\text { temperaturas }\end{array}$ \\
\hline & $\begin{array}{l}\text { Cálculo de gasto } \\
\text { metabólico mediante } \\
\text { calorimetría indirecta a } \\
22,28,32 \text { y } 35^{\circ} \mathrm{C}\end{array}$ & & $\begin{array}{l}\text { La menor tasa GMR/GMB se } \\
\text { mostró a temperatura ambiental } \\
\text { de } 32^{\circ} \mathrm{C}\end{array}$ \\
\hline $\begin{array}{l}\text { James et al, } \\
2017\end{array}$ & $\begin{array}{l}\text { Revisión sistemática } \\
4 \text { estudios }\end{array}$ & $\begin{array}{l}\text { Recomendación nivel 5, } \\
\text { opinión de expertos, } \\
\text { basada en los estudios de } \\
\text { Bishop y Latenser }\end{array}$ & $\begin{array}{l}\text { Prevención de la hipotermia } \\
\text { mediante habitación de } \mathrm{UCl} a \\
28-32^{\circ} \mathrm{C} \text {, fluidos calientes, } \\
\text { mantas calientes y aire } \\
\text { inspirado caliente }\end{array}$ \\
\hline Rizzo et al, 2017 & $\begin{array}{l}\text { Revisión sistemática } \\
15 \text { estudios de } 1972 \text { a } \\
2013\end{array}$ & $\begin{array}{l}\text { Temperaturas de } 22 \text { a } 40^{\circ} \mathrm{C} \\
\text { en el cuidado de pacientes } \\
\text { quemados en UCl y en } \\
\text { quirófano }\end{array}$ & $\begin{array}{l}\text { Necesidad de actualización de } \\
\text { los estudios de calentamiento y } \\
\text { temperatura ambiental. } \\
\text { Necesarios estudios de } \\
\text { hipotermia moderada }\end{array}$ \\
\hline
\end{tabular}

Para disminuir la respuesta hipermetabólica, como medidas no farmacológicas, se puede actuar elevando la temperatura ambiental. Kelemen et al ${ }^{(25)}$ comprobaron que el rango de temperatura ambiental de $30^{\circ} \mathrm{C}$ a $32^{\circ} \mathrm{C}$ reduciría el hipermetabolismo en pacientes con quemaduras desde el $20 \%$ de SCQ, alcanzando una disminución de demanda energética del $22 \%$ frente a una temperatura ambiental de $22^{\circ} \mathrm{C}$. Temperaturas ambientales superiores a $32^{\circ} \mathrm{C}$ ya no aportarían este beneficio. Existiendo relación entre mayor superficie corporal quemada y una demanda de mayor temperatura ${ }^{(25)}$.

\section{Calentamiento externo activo}

El calentamiento externo activo se obtiene mediante la aplicación directa de calor sobre la superficie del cuerpo. La utilización de aire convectivo como método de calentamiento externo activo ha sido estudiada principalmente en el ámbito quirúrgico y del cuidado de pacientes críticos ${ }^{(26)}$. Su aplicación se muestra efectiva para alcanzar la normotermia partiendo desde una temperatura corporal de $32^{\circ} \mathrm{C}$. Mediante la aplicación de aire convectivo se alcanza una tasa de $1^{\circ} \mathrm{C}$ mayor de calentamiento frente a otros métodos ${ }^{(27)}$.

\section{DISCUSIÓN}

El paciente quemado precisa mantener una temperatura corporal más elevada debido al aumento del gasto energético. El mantenimiento de una temperatura ambiental elevada disminuye la respuesta hipermetabólica y por tanto el consumo energético. Las recomendaciones actuales se basan en las investigaciones de Wilmore, que estudió la repercusión metabólica de la temperatura ambiental elevada aplicada como método de recalentamiento externo pasivo.

Como se observa en la Tabla 1, existe un rango de temperatura considerado como seguro, pero no hay una delimitación exacta de una temperatura óptima de referencia para el paciente quemado. Al mismo tiempo, en la valoración secundaria, es necesario 
estimar los efectos del calentamiento externo en relación con factores como la ansiedad, el dolor o el disconfort.

Se observó en una Unidad de Quemados que la temperatura media de los pacientes al final de fase aguda se situó en torno a los $37^{\circ} \mathrm{C}$, cuando se esperan temperaturas cercanas a los $38^{\circ} \mathrm{C}$, hecho que hace necesario reflexionar sobre las medidas de calentamiento, y si la temperatura corporal de origen no infeccioso es el mejor valor de referencia para el mantenimiento de dichas medidas ${ }^{(5)}$. En contraposición, se ha observado que en los pacientes en edad pediátrica, no se desarrolla esta respuesta hipertérmica como en los adultos ${ }^{(28)}$.

La temperatura ambiental elevada tiene efectos desfavorables, como un aumento de proliferación y crecimiento de microorganismos ${ }^{(29)}$ o estrés por calor. El mantenimiento de la normotermia únicamente mediante recalentamiento externo pasivo a temperaturas elevadas crea ambientes de trabajo hostiles. Se precisaría de una intervención mediante la combinación de métodos de calentamiento externo pasivo que pueda disminuir la temperatura ambiental de la habitación y disminuir el disconfort de pacientes y trabajadores.

Varios autores y guías recomiendan el uso conjunto de medidas de calentamiento como aire caliente inspirado o mantas calientes $(9,12,16,27)$, pero no hay especificaciones precisas sobre el uso de un tipo de calentamiento externo activo, además existe variabilidad en cuanto al momento de aplicación y su disponibilidad. Este método es de uso limitado en pacientes quemados, ya que mientras se realizan actuaciones de cuidado de la piel, no puede aplicarse con comodidad ni eficacia.

Existen experiencias con métodos de calentamiento interno activo como la utilización de un catéter intravascular destinado a la termorregulación ya que produce una rápida respuesta tanto en el calentamiento como en el enfriamiento de pacientes frente a otros métodos. Su uso está limitado en el tiempo debido al riesgo de trombosis intravascular ${ }^{(30)}$, por lo que no se puede extender el tratamiento más de 72 horas.

Un método de calentamiento externo activo que emplea tasas y rango de calentamiento y enfriamiento mediante la aplicación cutánea de parches de gel como Articsun $5000 \AA$, cuya aplicación está contraindicada sobre piel con ulceras, heridas o quemaduras, lo que impide su utilización en el caso de pacientes con quemaduras extensas.

Los métodos de calentamiento externo activo pueden mejorar el mantenimiento de la temperatura y la pérdida de calor sin crear ambientes adversos para los profesionales sanitarios durante la atención, optimizando además la regulación térmica del paciente quemado. La adecuación de los métodos de calentamiento podría lograr una mejora del confort térmico del paciente y de los trabajadores en la Unidad de Quemados consiguiendo una disminución la temperatura ambiental elevada.

\section{CONCLUSIONES}

El paciente gran quemado precisa una temperatura corporal superior a $37-37,5^{\circ} \mathrm{C}$ alcanzado los $38,5^{\circ} \mathrm{C}$, para evitar la temperatura crítica y disminuir el gasto energético, controlando el estado hipercatabólico. 
La temperatura ambiental recomendada en las unidades de grandes quemados se sitúa entre 28 a $33^{\circ} \mathrm{C}$. La aplicación de temperaturas ambientales elevadas crea ambientes hostiles para los trabajadores y pacientes, por lo que su aplicación efectiva en estas unidades es variable y precisa una adaptación y equilibrio entre las necesidades del paciente y las condiciones de los trabajadores.

Debe revisarse la recomendación de calentamiento mediante temperatura ambiental elevada a través del estudio de la inclusión de métodos de calentamiento externo activo en pacientes quemados.

\section{REFERENCIAS}

1. OMS. Quemaduras. 2018. Quemaduras. https://www.who.int/es/news-room/factsheets/detail/burns. Accedido diciembre 30, 2019.

2. European-Burn-Association. European Practice Guidelines for Burn Care. Minimum level of Burn Care Provision in Europe. Barcelona; 2017. www.eba2019.org. Accedido enero 4, 2020.

3. Weaver MD, Rittenberger JC, Patterson PD, et al. Risk factors for hypothermia in ems-treated burn patients. Prehospital Emerg Care. 2014;18(3):335-341. doi:10.3109/10903127.2013.864354

4. Sommerhalder C, Blears E, Murton AJ, Porter C, Finnerty C, Herndon DN. Current problems in burn hypermetabolism. Curr Probl Surg. 2020;57(1):100709. doi: 10.1016/j.cpsurg.2019.100709

5. Alonso-Fernández JM, Lorente-González P, Pérez-Munguía L, Cartón-Manrique AM, Peñas-Raigoso MC, Martín-Ferreira T. Analysis of hypothermia through the acute phase in major burns patients: Nursing care. Enferm Intensiva. 2019. doi: 10.1016/j.enfi.2019.05.002

6. Hostler D, Weaver MD, Ziembicki JA, et al. Admission temperature and survival in patients admitted to burn centers. J Burn Care Res. 2013;34(5):498-6. doi:10.1097/BCR.0b013e3182a231fb

7. Singer AJ, Taira BR, Thode $\mathrm{HC}$, et al. The association between hypothermia, prehospital cooling, and mortality in burn victims. Acad Emerg Med. 2010;17(4):456459. doi:10.1111/j.1553-2712.2010. 00702.x

8. Lang TC, Zhao R, Kim A, et al. A Critical Update of the Assessment and Acute Management of Patients with Severe Burns. Adv Wound Care. 2019;8(12):607-633. doi:10.1089/wound.2019.0963

9. Gaikwad M. Evidence Summary. Burns Units (Hypothermia): Prevention and Managemen Treatment. Joanna Briggs Inst EBP Database, JBI@Ovid. 2016; JBI15718. 10. Wilmore DW, Mason AD, Johnson DW, Pruitt BA. Effect of ambient temperature on heat production and heat loss in burn patients. J Appl Physiol. 1975;38(4):593-597. doi:10.1152/jappl.1975.38.4.593

11. Pham TN, Bettencourt AP, Bozinko GM, et al. Advanved Burn Life Support Course. Provider manual. 2018 Update. ChicGO; 2018.

12. Icaza I. Guía Práctica Clínica Sociedad De Quemaduras. Burns. 2016;42(Xxxx):176. doi: 10.1016/j.burns.2016.05.013

13. Cancio LC. Initial Assessment and Fluid Resuscitation of Burn Patients. Surg Clin North Am. 2014;94(4):741-754. doi: 10.1016/j.suc.2014.05.003

14. Al-Mousawi AM, Mecott-Rivera GA, Jeschke MG, Herndon DN. Burn Teams and Burn Centers: The Importance of a Comprehensive Team Approach to Burn Care. Clin Plast Surg. 2009;36(4):547-554. doi: 10.1016/j.cps.2009.05.015 
15. Pruskowski KA, Rizzo JA, Shields BA, et al. A Survey of Temperature Management Practices among Burn Centers in North America. J Burn Care Res. 2018;39(4):612-617. doi:10.1093/jbcr/irx034

16. Bishop S, Maguire S. Anaesthesia and intensive care for major burns. Contin Educ Anaesthesia, Crit Care Pain. 2012;12(3):118-122. doi:10.1093/bjaceaccp/mks001

17. Fagan SP, Bilodeau M-L, Goverman J. Burn Intensive Care. Surg Clin North Am. 2014;94(4):765-779. doi: 10.1016/j.suc.2014.05.004

18. Stanojcic M, Abdullahi A, Rehou S, Parousis A, Jeschke MG. Pathophysiological Response to Burn Injury in Adults. Ann Surg. 2018;267(3):576-584. doi:10.1097/SLA.0000000000002097

19. Lowe JM, Brody RA. Nutrition Management of Major Burn Injuries. Top Clin Nutr. 2019;34(2):161-171. doi:10.1097/TIN.0000000000000171

20. Prins A. Review: Nutritional management of the burn patient. S Afr J Clin Nutr. 2009;22(1):9-15.

21. Leung J, Ridley EJ, Cleland H, Ihle JF, Paul E, King SJ. Predictive energy equations are inaccurate for determining energy expenditure in adult burn injury: a retrospective observational study. ANZ J Surg. 2019;89(5):578-583. doi:10.1111/ans.15119

22. Zawacki BE, Spitzer KW, Mason AD, Johns LA. Does increased evaporative water loss cause hypermetabolism in burned patients? Ann Surg. 1970;171(2):236-240. doi:10.1097/00000658-197002000-00011

23. Busche MN, Roettger A, Herold C, Vogt PM, Rennekampff H-O. Evaporative Water Loss in Superficial to Full Thickness Burns. Ann Plast Surg. 2016;77(4):401-405. doi:10.1097/SAP.0000000000000845

24. James S, Campbell J. Temperature Management (Burns): Intensive Care Unit. Joanna Briggs Inst EBP Database, JBI@Ovid 2019; 2017; JBI13577.

25. Kelemen JJ, Cioffi WG, Mason AD, Mozingo DW, Mcmanus WF, Pruitt BA. Effect of Ambient Temperature on Metabolic Rate After Thermal Injury. Ann Surg. 1996;223(4):406-412. doi: 10.1097/00000658-199604000-00009

26. Steele JE, Atkins JL VM. Factors at scene in transfer related to the develoment of hypothermia in major burns. Ann Burns Fire Disasters. 2016;29(2):103-107. https://www.ncbi.nlm.nih.gov/pmc/articles/PMC5286989/pdf/Ann-Burns-and-Fire-

Disasters-29-103.pdf. Accedido marzo 8, 2019.

27. Latenser BA. Critical care of the burn patient: The first 48 hours. Crit Care Med. 2009;37(10):2819-2826. doi:10.1097/CCM.0b013e3181b3a08f

28. McEntire SJ, Chinkes DL, Herndon DN, Suman OE. Temperature Responses in Severely Burned Children During Exercise in a Hot Environment. J Burn Care Res. 2010;31(4):624-630. doi:10.1097/BCR.0b013e3181e4ca14

29. Rizzo JA, Rowan MP, Driscoll IR, Chan RK, Chung KK. Perioperative Temperature Management during Burn Care. J Burn Care Res. 2017;38(1):e277-e283. doi:10.1097/BCR.0000000000000371

30. Davis JS, Rodriguez LI, Quintana OD, et al. Use of a Warming Catheter to Achieve Normothermia in Large Burns. J Burn Care Res. 2013;34(1):191-195. doi:10.1097/BCR.0b013e31826c32a2 
